\title{
Effective Online Power Management with Adaptive Interplay of DVS and DPM for Embedded Real-time System
}

\author{
Gang Chen \\ Kai Huang \\ Jia Huang \\ Christian Buckl \\ Alois Knoll \\ TU Munich, Germany \\ TU Munich, Germany \\ fortiss $\mathrm{GmbH}$, Germany \\ fortiss $\mathrm{GmbH}$, Germany \\ TU Munich, Germany \\ cheng@in.tum.de \\ huangk@in.tum.de \\ huang@fortiss.org \\ buckl@fortiss.org \\ knoll@in.tum.de
}

\begin{abstract}
Effective power management is an important design concern for modern embedded systems. In this paper, we present an effective framework to integrate both DVS and DPM to optimize the overall energy consumption. We propose an online algorithm to determine the optimal operating frequency and mode transition of a processor based on the runtime workload. Our algorithm runs in $O(n)$ time, where $n$ is the number of the events stored in the system buffer. A feasibility analysis is also presented, which serves as a criteria for setting the system buffer as well as runtime schedulabilty check. The evaluations with specifications of two commercial processors show that our algorithm is more energy-efficient compared to existing schemes in the literature.
\end{abstract}

\section{INTRODUCTION}

Energy efficiency has become one of the major goals in embedded system design. Using appropriate power management techniques, the lifetime for battery-operated systems could be extended and the heat dissipation could be decreased, lowering the requirement for expensive packaging and cooling technology. Power consumption of processors mainly comes from dynamic power consumption due to switching activity and static power consumption due to the leakage current [12]. In micrometer CMOS technology, dynamic power dominates power consumption of processors. As CMOS technologies are shifting toward sub-micron domains, the static power increases exponentially and becomes comparable or even greater than dynamic power.

To reduce dynamic power and static power consumption, two main mechanisms can be employed, i.e., Dynamic Voltage Frequency Scaling (DVS) and Dynamic Power Management (DPM), respectively. DVS reduces the dynamic power consumption by dynamically adjusting voltage and frequency of a processor. The disadvantage of this technique is the lack of means to reduce static power consumption. On the other hand, DPM reduces the static power by switching the processor to a sleep mode with low static power consumption. The limitations of the pure DPM are: (1) dynamic power consumption is not considered when making the DPM-related decisions; (2) mode-switching in processor causes additional energy and latency penalty. Actually, it is worthwhile to switch the processor to sleep mode only when the idle interval is longer than a certain threshold called break-even time.
DVS and DPM outperform each other in different workload and architecture configurations [8]. Concerning DVS, lower frequencies result in lower dynamic power consumption, which however prolong the task execution time and shorten idle intervals. Therefore, DVS techniques in general limit the opportunities of reducing static power. On the other hand, although running the system at higher frequencies can create longer sleep intervals and reduce more static power, DPM will cost more dynamic power and mode-switch overheads. In principle, DVS and DPM counteract each other with respect to energy reduction. The motivation of our work is to integrate DVS and DPM and find the best trade-off to reduce the total energy consumption.

This paper presents a framework that considers the tradeoff between DPM and DVS and minimizes the overall energy consumption for hard real-time tasks. The core of the framework is an online algorithm that dynamically determines the optimal processor speed and activation time instants based on the runtime workload. Our algorithm is in $O(n)$ complexity while it can take into account the break-even time and available frequency bounds of the processor. By considering the integration of DVS and DPM, our framework yields significant energy reduction compared to the related work in the literature, using specifications from two commercial processor cores. We also provide a schedulability analysis of our algorithm, which serves as a criteria for setting the system buffer as well as runtime schedulabilty check.

The rest of paper is organized as follows: Section II reviews related work in the literature. Section III presents basic models and the definition of studied problem. Section IV describes our algorithm and the corresponding feasibility analysis. Power management scheme based on our algorithm is depicted in Section V. Experimental evaluation is presented in Section VI and Section VII concludes the paper.

\section{RELATED WORK}

A lot of researchers have explored DVS or DPM on realtime embedded system [1], [2], [9], [11], [22]. However, only a few have considered the combination of both DPM and DVS. Chen and Kuo [4] propose to execute tasks at a certain speed and control the procrastination of real-time tasks. By accumulating the execution of the procrastinated real-time tasks 
in a busy interval, the processor can switch into sleep mode to reduce the energy. The work in [7] presents an algorithm on the interplay DVS and DPM to optimize the system-wide energy for frame-based real-time embedded applications. The algorithm requires that the period of an application is equal to its deadline. Based on the concept of device forbidden regions(DFRs), an algorithm is proposed in [6] to determine the optimal processor speed as well as switching decisions of a system to reduce the system-wide energy for periodic real-time tasks. Taking a set of well-known existing(DVS and DPM) policies, authors in [3] employ machine-learning mechanisms to adaptively determine a suitable policy according to the runtime workload.

Most of the above research on integration of DVS and DPM requires special patterns of event arrivals, such as periodic realtime events [4], or frame-based events with a period equal to its deadline [6], [7]. However, in practice, this precise timing information of event arrivals might not be known in advance, since the arrival time information depends on many nonfunctional factors, e.g., environmental impacts. To model the irregular events, Real-time Calculus (RTC) is proposed by Thiele et al. [19]. In [16], Maxiaguine et al. apply Real-time Calculus within DVS context and compute a safe frequency at periodic intervals with a predefined length to prevent buffer overflow of a system. Huang et al. [10] and Lampka et al. [13] proposed online DPM by predicting a tighter trace bound with different prediction algorithms, procrastinating the processing of arrived events as late as possible. The schedulability for online DVS algorithms is explored when the event arrivals are constrained by a given upper arrive curve in [5], [17]. In this paper, we propose a power management framework on the interplay of DVS and DPM for event streams with irregular arrival patterns. In this framework, a online algorithm, namely Optimal Workload-Aware Algorithm (OWAA), is proposed to determine an optimal point of switching the processor from sleep mode to active mode with an optimal active frequency. Arrival-curve model is adopted to predict the worst-case idle interval to decide whether the processor is switched into sleep mode.

\section{Models And Problem}

This section describes the system models used in this paper, which is the basis of our work.

\section{A. Power Model}

The power model in [5] is adopted in this article. The power consumption can be described as follows:

$P(f)=P_{\text {sta }}+H\left(P_{\text {ind }}+P_{d}\right)=P_{\text {sta }}+H\left(P_{\text {ind }}+C_{\text {ef }} f^{\gamma}\right)$ where $P_{s t a}, P_{i n d}$, and $P_{d}$ represent static power, frequencyindependence power, and frequency-dependence power, respectively. $H$ is set to 1 and 0 when the processor is in active and sleep modes. Thus, $P_{\text {sta }}$ can be seen as the sleep power. Moreover, $C_{e f}$ and $\gamma$ respectively refer to the switch capacitance and the dynamic power exponent, which are system-dependent constants. As the existence of $P_{\text {ind }}$, there is a critical frequency $f_{\text {crit }}=\sqrt[\gamma]{\frac{P_{\text {ind }}}{C_{e f}(\gamma-1)}}$, which indicates that executing at frequency $f_{\text {crit }}$ is more energy-efficient than frequencies lower than $f_{\text {crit }}$.

Considering the overhead of switching the processor between active mode and sleep mode, the processor breakeven time $T_{B E T}$ indicates the minimum time length that the processor should stay at sleep mode. If the interval at which the processor can stay at sleep mode is smaller than $T_{B E T}$, the mode-switch mode overheads are larger than the energy saving. Therefore, mode-switch is not worthy. The break-even time $T_{B E T}$ can be defined as follows:

$$
T_{B E T}=\max \left(t_{s w}, \frac{E_{s w}-P_{s} \cdot t_{s w}}{P_{i}-P_{s}}\right)
$$

Where $t_{s w}$ and $E_{s w}$ denote the total state transition time and energy overhead, respectively. $P_{i}$ and $P_{s}$ respectively represent the idle power and sleep power.

\section{B. Event Model}

This paper considers events that could come irregularly. To model such events, the concept of arrival curve, which originated from Network Calculus [15] and Real-time Calculus(RTC)[14], [19], is adopted. In this context, a cumulative function $R(s, t)$ is used to describe an event stream in a system, which is defined as the number of events arriving in the time interval $[s, t)$. In any time interval of length $\Delta$, event stream $R$ can be characterized by an arrival curve $\alpha(\Delta)$ :

$$
R(t+\Delta)-R(t) \leq \alpha(\Delta), \forall t, \Delta \geq 0
$$

with $\alpha(\Delta)=0$ for $\Delta \leq 0$.

Arbitrary event arrival patterns are specified by means of arrival curves [19]. In this paper, we just consider one event stream, assuming the events in stream have the same execution time $C$ at maximum frequency $f_{\max }$ and the same related deadline $D$. Besides, at each time instant $t$, a trace of past events could be used to predict a tight bound $\mu(\Delta, t)$ for event stream $R$. See [10], [13] for details.

\section{Problem Statement}

Given are a processor $P$, an event stream $R$ characterized by arrival curve $\alpha$, and an energy model $E$. We are to design a power management scheme, that should decide when to conduct the system mode-switch (sleep, idle, or active) and how to schedule the frequency, such that (a) all the events can complete before their deadlines, (b) the frequencies produced by scheduler are within processors' available frequency bounds, and (c) the overall energy consumption of processors is minimized.

Note that in this work, we focus on the processor whose overhead for frequency scaling is small enough and can be neglected compared to the worst case execution time $C$. Therefore, we don't consider the processor's overhead for frequency scaling.

\section{OUR Algorithm}

In order to interplay DVS and DPM, a scheduler in general has to compute: (a) the switching time instant and the active frequency to turn the processor from sleep mode to active mode, (b) the time instant and the value of the frequency to 


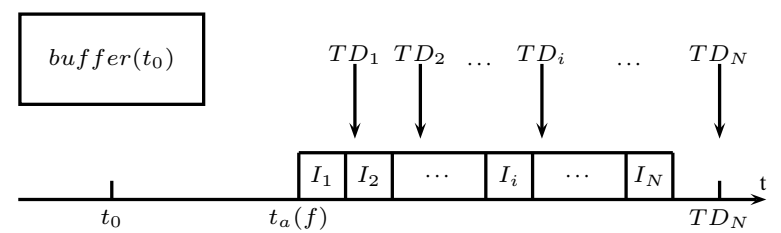

Fig. 1. Graphical View of Event Processing

scale the frequency when in active mode, and (c) when to turn the processor back to sleep mode. Therefore, we have to deal with activation decision, frequency scaling decision, and deactivation decision. This section presents an online algorithm that computes the activation decisions when the processor is in sleep mode and frequency scaling decisions when the processor is in active mode. System deactivation is considered in Section V-A. Combining DVS and DPM, our algorithm looks for the minimum energy consumption based on the runtime workload. The algorithm is triggered when the runtime workload is updated and it renews the previous computed activation and frequency scaling decisions. The algorithm determines an optimal execution frequency $f$ as well as an activation time instant $t_{a}$ by which the overall energy consumption is minimized upon the current workload, taking into account the maximum speed that the processor can provide. Analysis is also presented to depict the feasibility and complexity of the algorithm.

\section{A. Optimal Workload-Aware Algorithm}

Fig. 1 illustrates the event processing. Assume at current time instant $t_{0}$, the set of unprocessed events, $e_{1}, e_{2}, \ldots, e_{N}$, is denoted as buffer $\left(t_{0}\right)$. Note that all events are sorted by their arrival time in system buffer, and the event with earliest arrival time is processed first. $a_{i}, D$, and $C_{i}$ are respectively the arrival time, relative deadline, and remaining worst-case execution time of event $e_{i}$. $C$ is the worst-case execution time, thus $C_{i} \leq C . T_{a}(f)$ denotes the latest activation time of the processor, under which all events in buffer $\left(t_{0}\right)$ are guaranteed to satisfy their deadlines, using frequency $f . I_{i}$ represents the interval of processing event $e_{i}$, which can be computed by $I_{i}=\frac{C_{i}}{f}$, while $T D_{i}$ represents the absolute deadline of $e_{i}$. To guarantee all events in buffer $\left(t_{0}\right)$ can be processed before their deadlines, the active time $T_{a}(f)$ and the frequency $f$ should meet the following constraint:

$$
T_{a}(f)+\frac{\sum_{j=1}^{i} C_{j}}{f} \leq a_{i}+D, \forall i \in N
$$

Thus the latest activation time $T_{a}(f)$ can be calculated based on buffer $\left(t_{0}\right)$ and frequency $f$ :

$$
T_{a}(f)=D+\min _{i=1}^{N}\left(a_{i}-\frac{\sum_{j=1}^{i} C_{j}}{f}\right)
$$

The above equation shows that the time instant for activating the processor and the operating frequency $f$ are correlated. The slower frequency at which the processor runs, the earlier the processor needs to be turned on. For brevity, we define:

Def. 1: $T_{a}(f, k)$ represents the activation time instant when the processor runs at frequency $f$ and $a_{i}-\frac{\sum_{j=1}^{i} C_{j}}{f}$ with $i=$ $1, \ldots, N$ gets its minimum value at $i=k$. Then, $T_{a}(f, k)=$
$D+\left(a_{k}-\frac{\sum_{j=1}^{k} C_{j}}{f}\right)$.

When the processor turns on at $T_{a}(f, k)$ and runs at frequency $f$, event $e_{k}$ completes right on its deadline.

According to the power model, the energy consumption of processing all events in the system buffer is:

$$
E(k, f)=P(f) \frac{\sum_{j=1}^{N} C_{j}}{f}+P_{\text {sta } / \text { idle }}\left(T_{a}(f, k)-t_{0}\right)
$$

where, at time instant $t_{0}, P_{\text {sta }}$ idle is set to sleep power $P_{\text {sta }}$ when the processor is in sleep mode and idle power $P_{\text {idle }}$ when in active mode.

With Eqns. (1), (5), and (6), the energy consumption $E(k, f)$ can be computed as:

$$
\begin{aligned}
E(k, f)= & \left(P_{\text {sta }}+P_{\text {ind }}+C_{\text {ef }} f^{\gamma}\right) \frac{\sum_{j=1}^{N} C_{j}}{f} \\
& +P_{\text {sta } / \text { idle }}\left(D+a_{k}-\frac{\sum_{j=1}^{k} C_{j}}{f}-t_{0}\right) \\
= & C_{e f} \sum_{j=1}^{N} C_{j} f^{\gamma-1}+\left(\left(P_{\text {sta }}+P_{\text {ind }}\right) \sum_{j=1}^{N} C_{j}\right. \\
& \left.-P_{\text {sta } / \text { idle }} \sum_{j=1}^{k} C_{j}\right) \frac{1}{f}+P_{\text {sta } / \text { idle }}\left(D+a_{k}-t_{0}\right)
\end{aligned}
$$

For a specific event $e_{k}, P_{\text {sta } / \text { idle }}\left(D+a_{k}-t_{0}\right)$ can be seen as a constant in $E(k, f)$. As $\gamma \geq 1$, frequency that minimizes $E(k, f)$ can be found by analyzing its first-order derivative. Optimal frequency $f_{\text {opt }}(k)$ for event $e_{k}$ can be given as:

$$
f_{\text {opt }}(k)=\sqrt[\gamma]{\frac{\left(P_{\text {sta }}+P_{\text {ind }}\right) \sum_{j=1}^{N} C_{j}-P_{\text {sta } / i d l e} \sum_{j=1}^{k} C_{j}}{C_{e f}(\gamma-1) \sum_{j=1}^{N} C_{j}}}
$$

By analyzing its first-order derivative, the following properties hold for any $\epsilon \geq 0$.

Prop. 1: $\forall f, f>f_{\text {opt }}(k), E\left(k, f_{\text {opt }}(k)\right) \leq E(k, f) \leq$ $E(k, f+\epsilon)$

Prop. 2: $\forall f, f<f_{\text {opt }}(k), E\left(k, f_{\text {opt }}(k)\right) \leq E(k, f) \leq$ $E(k, f-\epsilon)$

Further more, we can proof that $E(k, f)$ is strictly convex to $f$ only when $\gamma \geq 2$ holds by analyzing its second-order derivative.

For event $e_{k}$, there is a corresponding accepted frequency range $\left[f_{l}(k), f_{u}(k)\right]$, which makes $a_{i}-\frac{\sum_{j=1}^{i} C_{j}}{f}$ get its minimum value at event $e_{k}$. The accepted range for $e_{k}$ can be determined as follows.

$$
a_{k}-\frac{\sum_{j=1}^{k} C_{j}}{f} \leq a_{i}-\frac{\sum_{j=1}^{i} C_{j}}{f}, i \neq k
$$

For the case of $i \geq k+1$, there is $a_{i} \geq a_{k}$, then

$$
f \geq \frac{\sum_{j=1}^{i} C_{j}-\sum_{j=1}^{k} C_{j}}{a_{i}-a_{k}}
$$

For the case of $i \leq k-1$, there is $a_{i} \leq a_{k}$, then

$$
f \leq \frac{\sum_{j=1}^{i} C_{j}-\sum_{j=1}^{k} C_{j}}{a_{i}-a_{k}}
$$

To compute the accepted frequency range $\left[f_{l}(k), f_{u}(k)\right]$ for event $e_{k}$, the accepted frequency function $\operatorname{AF}\left(e_{k}, e_{i}\right)$ between $e_{k}$ and $e_{i}$ is defined as follows: 


$$
\text { Def. 2: } A F\left(e_{k}, e_{i}\right)= \begin{cases}+\infty & a_{i}=a_{k} \wedge i>k \\ -\infty & a_{i}=a_{k} \wedge i<k \\ \frac{\sum_{j=1}^{i} C_{j}-\sum_{j=1}^{k} C_{j}}{a_{i}-a_{k}} & \text { otherwise }\end{cases}
$$

Thus, with the frequency bounds $\left[f_{\min }, f_{\max }\right]$ that processor can provide, the accepted range $\left[f_{l}^{*}(k), f_{u}^{*}(k)\right]$ for event $e_{k}$ can be calculated as:

$$
\begin{aligned}
& f_{l}^{*}(k)= \begin{cases}\max _{i=k+1}^{N} A F\left(e_{k}, e_{i}\right) & 1 \leq k \leq N-1 \\
f_{\min } & k=N\end{cases} \\
& f_{u}^{*}(k)= \begin{cases}\min _{i=1}^{k-1} A F\left(e_{k}, e_{i}\right) & 2 \leq k \leq N \\
f_{\max } & k=1\end{cases}
\end{aligned}
$$

When the processor turns on at $T_{a}(f, k)$ and runs at frequency $f$, event $e_{k}$ completes right on its deadline $a_{k}+D$. Consider the worst case of turning on system at current time instant $t_{0}$. To handle the worst case, the frequency $f$ should be no less than the gate frequency $f_{g}\left(t_{0}, k\right)$.

$$
f_{g}\left(t_{0}, k\right)=\frac{\sum_{j=1}^{k} C_{j}}{a_{k}+D-t_{0}}
$$

Correspondingly, the accepted frequency $\left[f_{l}(k), f_{u}(k)\right]$ should be adjusted to:

$$
\begin{aligned}
& f_{l}(k)=\max \left(f_{l}^{*}(k), f_{g}\left(t_{0}, k\right), f_{\text {min }}\right) \\
& f_{u}(k)=\min \left(f_{u}^{*}(k), f_{\text {max }}\right)
\end{aligned}
$$

The case $f_{l}(k)>f_{u}(k)$ indicates that $T_{a}(k, f)$ is not acceptable for event $e_{k}$. Let $\bar{f}(k)$ denote the frequency that minimizes the energy consumption when $T_{a}(k, f)$ is accepted in event $e_{k}$. From Prop. 1 and Prop. $2, \bar{f}(k)$ can be determined by considering three possible scenarios:

$$
\bar{f}(k)= \begin{cases}f_{\text {opt }}(k) & \text { if } f_{l}(k) \leq f_{\text {opt }}(k) \leq f_{u}(k) \\ f_{l}(k) & \text { if } f_{\text {opt }}(k) \leq f_{l}(k) \leq f_{u}(k) \\ f_{u}(k) & \text { if } f_{l}(k) \leq f_{u}(k) \leq f_{\text {opt }}(k)\end{cases}
$$

The above derivation finds an optimal frequency $\bar{f}(k)$ for each event $e_{k}$ in the unprocessed workload. $\bar{f}(k)$ is the local optimal frequency when $T_{a}(k, f)$ is accepted in event $e_{k}$. To determine the global optimal frequency, it is essential to consider all the local optimal frequency for every acceptable event currently stored in the system buffer and compare their energy consumption to select a global optimal accepted event $e_{o p t}$. Thus, at time instant $t_{0}$, the global optimal frequency $\bar{f}_{\text {gopt }}$ and corresponding activation time $\overline{t_{a}}$ can be calculated as follows:

$$
\begin{aligned}
& \bar{f}_{\text {gopt }}=\bar{f}\left(e_{\text {opt }}\right) \\
& \overline{t_{a}}=D+a_{e_{o p t}}-\frac{\sum_{j=1}^{e_{o p t}} C_{j}}{\bar{f}_{\text {gopt }}}
\end{aligned}
$$

where $e_{\text {opt }}=\left\{k \mid E(k, \bar{f}(k))=\min _{i=1}^{N} E(i, \bar{f}(i)), k=\right.$ $1, N\}$.

Algo. 1 traces the current workload and generates an optimal decision $\left(\overline{f_{g o p t}}, \overline{t_{a}}\right)$ which minimizes the energy consumption. Algo. 1 is triggered when workload is changed, i.e., at the arrival of every new event (Lines 1-9) and completion of processing for every event (Lines 10-14). The update accepted frequency routine (Line 6, Line 11) is used to refresh the accepted frequency in Eqns. (11) and (12) for all events in the back-

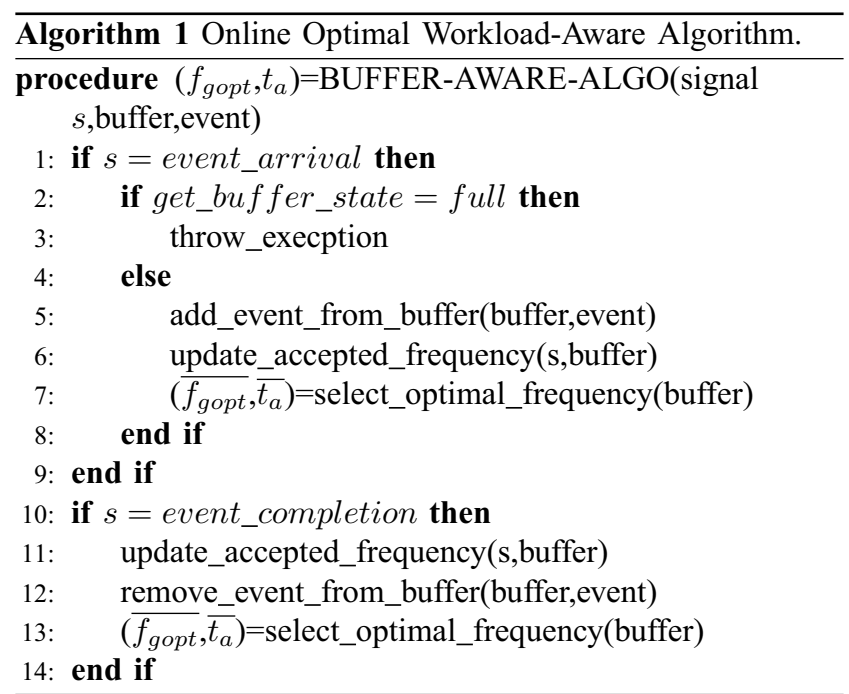

log. The select_optimal_frequency routine computes the local optimal frequency $\bar{f}$ of each event using Eqn. (13), then selects the optimum using Eqns. 14 and 15. The complexity of update_accepted_frequency and select_optimal_frequency is $O(N)$ (see Section IV-C). In our approach, Algo. 1 is used to generate optimal activation decisions when the processor is in sleep mode and frequency scaling decisions when the processor is in active mode.

\section{B. Algorithm Feasibility Analysis}

This section presents a schedulability analysis of our algorithm. We prove that our algorithm can guarantee that (a) all events in the system backlog can be processed within their deadlines; (b) under certain condition, the chosen frequency $\overline{f_{\text {gopt }}}$ does not exceed the maximal available frequency $f_{\max }$ and the activation time $\overline{t_{a}}$ is no earlier than at current time instant $t_{0}$.

Lem. 1: Turning on the processor at time instant $\overline{t_{a}}$ with frequency $\bar{f}_{\text {gopt }}$ computed by Algo. 1 will cause no deadline violation of the unprocessed events in the system buffer.

Proof: With Eqns. (15) and (5), we can get:

$$
\overline{t_{a}}=D+\min _{i=1}^{N}\left(a_{i}-\frac{\sum_{j=1}^{i} C_{j}}{\bar{f}_{\text {gopt }}}\right) \leq D+\left(a_{i}-\frac{\sum_{j=1}^{i} C_{j}}{\bar{f}_{\text {gopt }}}\right) .
$$

Thus

$$
\overline{t_{a}}+\frac{\sum_{j=1}^{i} C_{j}}{\bar{f}_{\text {gopt }}} \leq D+a_{i}, \forall e_{i}
$$

holds. The left side of Eqn. (16) is the completion time of $e_{i}$ and the right side is the absolute deadline of $e_{i}$. The inequality shows that all events complete no later than their deadlines.

Algo. 1 is triggered only when the workload is changed, i.e., at the time instant of the arrival and completion of an event. Thus, the feasibility analysis can be divided into two cases, i.e., new event arrival and event completion scenarios. Consider the feasibility of the worst case of our algorithm, i.e., 


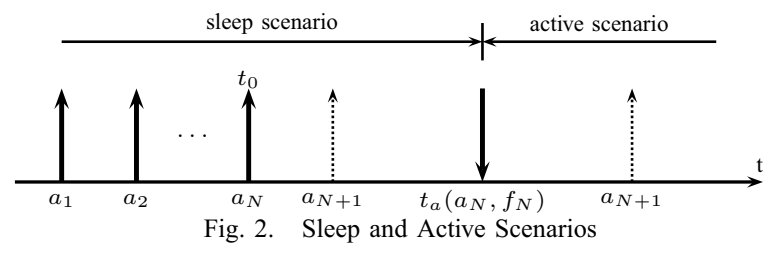

all events in the system buffer can be completed within their deadlines when the processor turns on at current time instant $t_{0}$ and executes at $f_{\max }$. The feasibility of the worst case can guarantee that, at any time instant when the scheduling decision is re-computed, the resulted activation time will be no earlier than the current time and the computed frequency is no larger than $f_{\max }$.

Lem. 2: At the time instant of event completion $t_{0}$, Algo. 1 is feasible.

Proof: Let $t_{p}$ be the last updating time instant before $t_{0}$ and $f_{p}$ be the updating frequency computed at $t_{p}$. According to Lem. 1, at time instant $t_{p}$, all events in buffer $\left(t_{p}\right)$ can be safely processed under $f_{p}$. During the interval $\left(t_{p}, t_{0}\right]$, the workload is decreased due to event completion. It is apparent that running system at $f_{p}$ and activating system at current time instant $t_{0}$ can guarantee the remaining events to complete before their deadlines. As $f_{\max } \geq f_{p}$, the worst case is also feasible.

Lem. 3: Define buf(t) as the number of events stored in the system buffer at time instant $t$ and $a_{N}$ as the arrival time instant of $N^{t h}$ event. If $\operatorname{buf}\left(a_{N}\right) \leq\left\lfloor\frac{D f_{\max }}{C}\right\rfloor$ holds, Algo. 1 can find a feasible frequency and active time at updating time instance $a_{N}$.

Proof: We divide the proof into two cases, i.e., new event arrives before and after activation time $t_{a}$. Lets call these two cases sleep scenario and active scenario, respectively. Let $t_{a}(t, f)$ denote the updated activation time at time instant $t$. Fig. 2 shows that at time instant $a_{N}$, the system buffer contains $N$ events with arrival time $a_{1}, \ldots, a_{N}$. Assume the arrival of new event $e_{N}$ triggers the processor to update the feasible frequency $f_{N}$ and new activation time $t_{a}\left(a_{N}, f_{N}\right)$. $a_{N+1}$ denotes the arrival time instant for next new event. Sleep scenario is the case of $a_{N+1}<t_{a}\left(a_{N}, f_{N}\right)$ while active scenario is the case of $a_{N+1} \geq t_{a}\left(a_{N}, f_{N}\right)$. What we need to prove is that, for both cases, the condition of buf $\left(a_{N+1}\right) \leq\left\lfloor\frac{D f_{\max }}{C}\right\rfloor$ can guarantee that Algo. 1 is feasible at updating time instant $a_{N+1}$.

- sleep scenario $\left(a_{N+1}<t_{a}\left(a_{N}, f_{N}\right)\right)$ :

For all events $e_{i}(i=1, \ldots, N)$, assume $a_{i}-\frac{\sum_{j=1}^{i} C_{j}}{f_{\max }}$ gets its minimal value at event $e_{p}$. According to Eqn. (5) and $f_{N} \leq$ $f_{\max }$, we have:

$t_{a}\left(a_{N+1}, f_{\max }\right)=\min \left(t_{a}\left(a_{N}, f_{\max }\right), D+a_{N+1}-\frac{\sum_{j=1}^{N+1} C_{j}}{f_{\max }}\right)$

$$
\begin{aligned}
t_{a}\left(a_{N}, f_{\text {max }}\right) & =D+a_{p}-\frac{\sum_{j=1}^{p} C_{j}}{f_{\max }} \geq D+a_{p}-\frac{\sum_{j=1}^{p} C_{j}}{f_{N}} \\
& \geq D+\min _{k=1}^{N}\left(a_{k}-\frac{\sum_{j=1}^{k} C_{j}}{f_{N}}\right)=t_{a}\left(a_{N}, f_{N}\right) \\
& >a_{N+1}
\end{aligned}
$$

As buf $\left(a_{N+1}\right) \leq\left\lfloor\frac{D f_{\max }}{C}\right\rfloor$ and $C_{j} \leq C$ hold, we can get:

$D+a_{N+1}-\frac{\sum_{j=1}^{N+1} C_{j}}{f_{\max }} \geq D+a_{N+1}-\frac{b u f\left(a_{N+1}\right) C}{f_{\max }} \geq a_{N+1}$

With Eqns. (17), (18), and (19), we have $t_{a}\left(a_{N+1}, f_{\max }\right) \geq$ $a_{N+1}$ which shows that at the next updating time instant $a_{N+1}$, we can always activate the processor not earlier than $a_{N+1}$ when the processor runs at the maximal frequency.

- active scenario $a_{N+1} \geq t_{a}\left(a_{N}, f_{N}\right)$ :

Let $t_{p}$ be the last updating time instant before $a_{N+1}$. Thus, $b u f\left(a_{N+1}\right)=b u f\left(t_{p}\right)+1$. Assume $f_{p}$ is the updating frequency at $t_{p}$. According to Lem. 1 , at time instant $t_{p}$, all events in buffer $\left(t_{p}\right)$ can be safely processed under $f_{p}$. Consider the worst case of active scenario, i.e., the processor turns on at $a_{N+1}$ and runs at $f_{\max }$. Running processor at $f_{p}$ and activating processor at current time instants $a_{N+1}$ can guarantee the first buf $\left(t_{p}\right)$ events in buffer $\left(a_{N+1}\right)$ to complete before their deadlines. Notes that $f_{p}$ cannot guarantee the last event $e_{N+1}$ to complete before its deadline. As $f_{\max } \geq f_{p}$, the first $b u f\left(t_{p}\right)$ events can also be safely processed under under $f_{\max }$.

For the last event $e_{N+1}$ in buffer $\left(a_{N+1}\right)$, the completion time $T C\left(e_{N+1}\right)$ under $f_{\max }$ can be computed as $T C\left(e_{N+1}\right)=a_{N+1}+\frac{\sum_{j=1}^{b u f\left(a_{N+1}\right)} C_{j}}{f_{\max }} \leq a_{N+1}+D$ with buf $\left(a_{N+1}\right) \leq\left\lfloor\frac{D f_{\max }}{C}\right\rfloor$. It shows that the worst case of active scenario is feasible if buf $\left(a_{N+1}\right) \leq\left\lfloor\frac{D f_{\max }}{C}\right\rfloor$ holds.

From above cases, the lemma holds.

Lem. 3 shows that our algorithm can accumulate at most $\left\lfloor\frac{D f_{\max }}{C}\right\rfloor$ unprocessed events in the system buffer. It offers a criterion for allocating the capacity of the system buffer. We can set the capacity of the system buffer as $\left\lfloor\frac{D f_{\max }}{C} \mid\right.$. If the system buffer overflows, it indicates that our online algorithm is not feasible. Otherwise, our algorithm is feasible. Based on Lem. 3, we provide a general feasible analysis for arbitrary arrival patterns.

Lem. 4: Given an event stream $R$ characterized by $\alpha$, Algo. 1 is feasible when $\alpha(D) \leq\left\lfloor\frac{D f_{\max }}{C} \mid\right.$ holds.

Proof: Assume at current time $t_{0}, N$ events with arrival time $a_{1}, a_{2}, \ldots, a_{N}$ are stored in buffer $\left(t_{0}\right)$. According to deadline constraints, $a_{1}+D \geq t_{0} \geq a_{n}$. Thus, buf $\left(t_{0}\right)=R\left(a_{n}\right)-R\left(a_{1}\right) \leq \alpha\left(a_{n}-a_{1}\right) \leq \alpha(D)$. With $\alpha(D) \leq\left\lfloor\frac{D f_{\max }}{C}\right\rfloor$ and Lem. 3, we know Algo. 1 is feasible.

\section{Algorithm Complexity Analysis}

The computational overhead of Algo. 1 comes from (a) refreshing the accepted frequency $\left[f_{l}(k), f_{u}(k)\right]$ (Eqns. (11) and (12)) and (b) computing the optimal decisions (Eqn. (14) and (15)). 


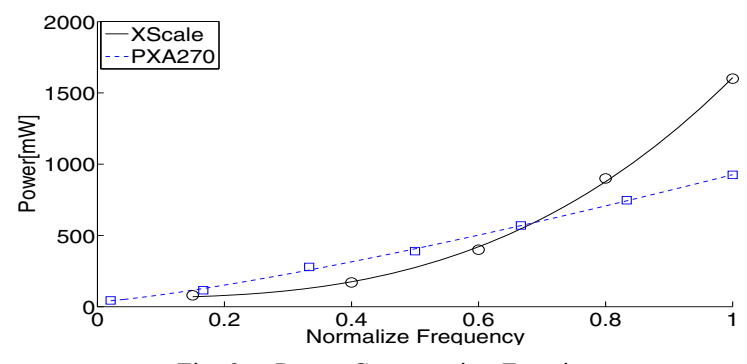

Fig. 3. Power Consumption Function

The accepted frequency $\left[f_{l}(k), f_{u}(k)\right]$ is refreshed at the point of adding new events to the system buffer or removing completed events from buffer. For brevity, let $\left[f_{l}^{*}(n, k), f_{u}^{*}(n, k)\right]$ be the accepted frequency bound determined by Eqns. (8) and (9) when buffer contains $n$ events. For the case of adding new event $e_{n+1}$ to buffer, $\left[f_{l}^{*}(n+\right.$ $\left.1, k), f_{u}^{*}(n+1, k)\right]$ can be refreshed by the following iterations:

$$
\begin{array}{r}
f_{l}^{*}(n+1, k)= \begin{cases}\max \left(f_{l}^{*}(n, k), A F\left(e_{k}, e_{n+1}\right)\right) & 1 \leq k \leq n \\
f_{\min } & k=n+1\end{cases} \\
f_{u}^{*}(n+1, k)= \begin{cases}f_{u}^{*}(n, k) & 1 \leq k \leq n \\
\min _{i=1}^{n} A F\left(e_{i}, e_{n+1}\right) & k=n+1\end{cases} \\
s_{u}^{*}(n+1, k)= \begin{cases}s_{u}^{*}(n, k) & 1 \leq k \leq n \\
\operatorname{smin}_{i=1}^{n} A F\left(e_{i}, e_{n+1}\right) & k=n+1\end{cases}
\end{array}
$$

Note that smin is the operation for getting the second minimum value in a group of numbers and $s_{u}^{*}(n, k)$ is used in case of removing completed event from the system buffer. smin can be executed integrated with min. Considering the gate frequency $f_{g}$ and the processor frequency bound $\left[f_{\min }, f_{\max }\right]$, the adjusted frequency could be determined by Eqns. (11) and (12). There are at most $n$ iterations in update_accepted_frequency routine when a new event is added into the system buffer.For the case of removing completed events, update_accepted_frequency routine also needs at most $n$ iterations. $\left[f_{l}^{*}(n-1, k), f_{u}^{*}(n-1, k)\right]$ could be similarly determined by the following iterations:

$$
\begin{aligned}
& f_{l}^{*}(n-1, k-1)=f_{l}^{*}(n, k) \\
& f_{u}^{*}(n-1, k-1)= \begin{cases}s_{u}^{*}(n, k) & f_{u}(n, k)=A F\left(e_{k}, e_{1}\right) \& k \geq 3 \\
f_{u}^{*}(n, k) & f_{u}(n, k) \neq A F\left(e_{k}, e_{1}\right) \& k \geq 3 \\
f_{\max } & k=2\end{cases}
\end{aligned}
$$

From above analysis, it can be concluded that the complexity of update_accepted_frequency is $O(n)$, where $n$ is the number of events stored in the system buffer.

From Eqns. (14) and (15), assuming there are $n$ accepted events in the system buffer, computing the optimal frequency $\overline{f_{o p t}}$ and activation time $\overline{t_{a}}$ also need $O(n)$ operations.

\section{Power Management Strategy}

A power management strategy involves solving the following two problems to save energy: (a) determine the activation time and the activation frequency when the processor is in sleep mode, and (b) determine the running frequency and the time to turn the processor back to sleep mode when the processor is in active mode. Meanwhile, the scheduler decisions must guarantee the real-time constraints and meet the frequency bounds.

\section{A. System Deactivation}

There are two scenarios that the system could switch to sleep mode, i.e., when the system buffer is empty and when the idle time interval $\overline{t_{a}}-t_{0}$ computed by Algo. 1 is longer than $T_{B E T}$. In the following, we consider these two scenarios individually.

Empty system buffer: Here we use an online DPM system deactivation scheme based on the dynamic counter technique proposed in [13]. To satisfy the required deadline $D$ and prevent system buffer with capacity $Q$ from overflow, the minimum service demand can be computed as follows:

$$
\beta(\Delta, t)=\max (\mu(\Delta, t)-Q, \mu(\Delta-D, t))
$$

where $\mu(\Delta, t)$ is the predicted bound of arrive curve determined by the dynamic counter. Then, the largest period $\tau$ that the processor can stay in sleep mode with predictive active frequency $f_{a}$ can be computed as:

$$
f_{a}(\Delta-\tau) \geq C \beta(\Delta, t)
$$

If $\tau$ is larger than the break-even time $T_{B E T}$, the processor should turn to sleep mode. Otherwise, the processor enters the idle mode. An interesting problem in this prediction is how to set the predictive frequency $f_{a}$. If $f_{a}$ is set to the maximum frequency $f_{\text {max }}$, processor can greedily explore the opportunity for energy saving, but a higher speed is required when the processor turns back to active mode. Moreover, in some scenarios, e.g., when the idle power of the processor is relative small and close to the sleep power, energy saving obtained by switching the system into sleep mode might be very limited. This method would produce pessimistic results, as the dynamic power consumption is the dominating part. In our approach, the critical speed $f_{\text {crit }}$ is selected as the predictive frequency to compute $\tau$. The choice generates better results for both processors used in our experiment.

Idle time interval $\overline{t_{a}}-t_{0} \geq T_{B E T}$ : Assume that at current updating time $t_{0},\left(\overline{f_{\text {gopt }}}, \overline{t_{a}}\right)$ is the newly computed optimal frequency and mode-switch point. The workload at time instant $t_{0}$ is denoted as $w\left(t_{0}\right)$. Consider any interval $\left[t_{0}, t_{0}+\Delta\right)$, the number of events arrived in this interval is upper-bounded by $\mu\left(\Delta, t_{0}\right)$. The future event $e_{\mu\left(\Delta, t_{0}\right)+1}$ arrives later than the time instant $t_{0}+\Delta$, i.e., $a_{\mu\left(\Delta, t_{0}\right)+1} \geq t_{0}+\Delta$. Let $t_{a}(t, f)$ denote the active time with active frequency $f$ at updating time instant $t$. To guarantee that the sleep time is larger than $T_{B E T}$ with arrival curve $\mu\left(\Delta, t_{0}\right), t_{a}\left(a_{\mu\left(\Delta, t_{0}\right)+1}, f_{\text {gopt }}\right)$ should be no less than $t_{0}+T_{B E T}$. According to Eqn. (5), the following constraint should be met for future event $e_{\mu}\left(\Delta, t_{0}\right)+1$.

$\inf _{\Delta \leq T_{B E T}}\left(D+a_{\mu\left(\Delta, t_{0}\right)+1}-\frac{w\left(t_{0}\right)+\left(\mu\left(\Delta, t_{0}\right)+1\right) C}{f_{\text {gopt }}}\right) \geq t_{0}+T_{B E T}$ 
With $a_{\mu\left(\Delta, t_{0}\right)+1} \geq t_{0}+\Delta$, we have:

$f_{\text {gopt }}\left(\Delta+D-\frac{w\left(t_{0}\right)}{f_{\text {gopt }}}-T_{B E T}\right) \geq\left(\mu\left(\Delta, t_{0}\right)+1\right) C \quad \Delta \leq T_{B E T}$

If Eqn. (28) holds, the processor can turn to sleep mode. Otherwise, the processor enters idle mode.

\section{B. Sytem Activation}

Once the processor enters sleep mode, it should go back to active mode with an appropriate frequency at a later moment for event processing. Further analysis is needed to find the optimal operating frequency and switching moment. On the one hand, it is beneficial to turn on processor as late as possible for the sake of static power reduction. The side-effect is however a higher execution frequency later in the active mode. On the other hand, an early wake-up would require a lower operating frequency and therefore reduce the dynamic power consumption. To resolve this contradiction, Algo. 1 is used to find the optimal switching point and operating frequency for the current workload. Algo. 1 works in an workload-triggered manner. Optimal decision is re-calculated when the workload is updated, e.g., at new event arrival and event completion. In sleep mode, the decision is also updated on arrival of new events.

Based on the switching point and active frequency determined by Algo. 1, a further concern is that the processor should stay in sleep mode long enough to achieve power reduction (i.e., the sleep time exceeds the break-even time $\left.T_{B E T}\right)$. As a result, Eqn. (10) should be adjusted as follows:

$$
f_{g}\left(t_{0}, k\right)=\frac{\sum_{j=1}^{k} C_{j}}{a_{k}+D-\max \left(t_{0}, t_{o f f}+T_{B E T}\right)}
$$

where $t_{o f f}$ is the time instant of turning off the processor.

Consider the scenario that a new event arrives at $t_{0}$, Algo. 1 generates the switch moment $t_{a}$ and operating frequency $f$. If no further event arrives before $t_{a}$, processor is turned on at $t_{a}$ with frequency $f$. Otherwise, the switching moment and execution frequency are updated at the time instant of the new event arrival. The feasibility of this activation strategy is already analyzed in Section IV-B (see Lem. 3).

\section{Frequency Selection On Active Mode}

When the processor enters active mode, Algo. 1 is used to compute the optimal frequency $\overline{f_{o p t}}$ and active time $\overline{t_{a}}$ to process the current workload. Here Algo. 1 works in a workload-triggered manner. The frequency is updated at the point of new event arrival and event completion. When $\overline{t_{a}}-t_{0} \geq T_{B E T}$ holds, a mode-switching decision is made. Otherwise, $\overline{f_{\text {opt }}}$ is applied immediately to process the events. When the system buffer is empty, the approach in Section V-A is applied to decide whether to switch the processor into sleep mode.

\section{Evaluation}

This section provides experimental evaluations for the proposed framework. We implement our algorithms in MATLAB and evaluate the performance by comparing with three existing on-line algorithms in literature [5], [13].

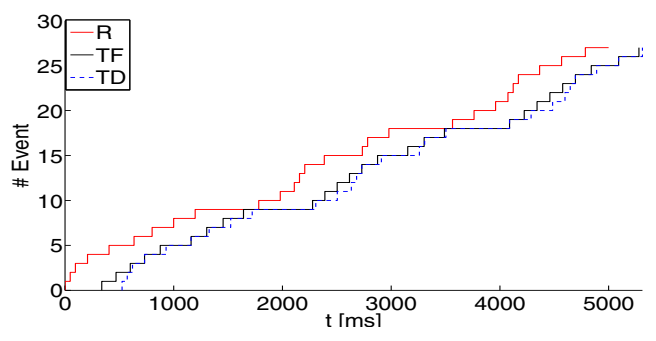

Fig. 4. Completion Time and Deadline

\begin{tabular}{l||rrrrrr}
\hline & S1 & S2 & S3 & S4 & S5 & S6 \\
\hline P & 198 & 102 & 283 & 239 & 148 & 114 \\
J & 387 & 70 & 269 & 222 & 91 & 13 \\
d & 48 & 45 & 58 & 65 & 78 & 0 \\
C & 35 & 11 & 45 & 38 & 20 & 15 \\
\hline
\end{tabular}

TABLE I

Parameters for Six Event Streams in [MS]

\section{A. Experimental Setup}

We adopt the PJD arrive pattern defined by parameters period $p$, maximum jitter $j$, and minimum event inter-arrival time $d$. The arrival curve for such models can be represented as $\alpha(\Delta)=\min \left(\left\lceil\frac{\Delta+j}{p}\right\rceil,\left\lceil\frac{\Delta}{d}\right\rceil\right)$. The specification of six event streams adopted in [17] is listed in Tab. I. The RTC/RTStoolbox [20] is used to generate the traces with different patterns. The worst-case execution time (WCET) at frequency $f_{\max }$ required for processing an event is also given in Tab. I. The WCETs for lower frequencies are normalized w.r.t the ratio to the $f_{\max }$. The relative deadline is set to the period times a deadline factor $\eta$.

We use the hardware parameters from Marvell PXA270 [18] and Intel XScale [21] in our experiments. The voltagefrequency levels supported by PXA270 and Intel XScale are listed in Tab. II. From those discrete operating points, we use the least square curve fitting to obtain the frequencypower function in the form of $a f^{\gamma}+b$ for each processor. In this case, the active power function of PXA270 and XScale can be written as $P_{a}(f)=35.09+891.24 f^{1.26}$ and $P_{a}(f)=63.58+1543.28 f^{2.87}$, respectively. The results are also depicted in Fig. 3. The idle power $P_{\text {idle }}$, sleep power $P_{\text {sta }}$, mode-switch energy overhead $E_{s w}$, mode-switch transition time overhead $t_{s w}$, and the critical speed $f_{c r i t}$ of the two processors are listed in Tab. III.

To evaluate the performance of our framework, we com-

\begin{tabular}{l|c||rrrrrrr}
\hline \multirow{3}{*}{ PXA270 } & $\mathrm{V}(V)$ & 1.55 & 1.45 & 1.35 & 1.25 & 1.15 & 0.9 & 0.85 \\
& $\mathrm{f}(M H z)$ & 624 & 520 & 416 & 312 & 208 & 104 & 13 \\
& $\mathrm{P}(m W)$ & 925 & 747 & 570 & 390 & 279 & 116 & 44.2 \\
\hline \multirow{3}{*}{ XScale } & $\mathrm{V}(\mathrm{V})$ & 1.8 & 1.6 & 1.3 & 1.0 & 0.75 & & \\
& $\mathrm{f}(M H z)$ & 1000 & 800 & 600 & 400 & 150 & & \\
& $\mathrm{P}(m W)$ & 1600 & 900 & 400 & 170 & 80 & & \\
\hline
\end{tabular}

TABLE II

Active Power Consumption for Processors PXA270 And XScale 


\begin{tabular}{l|ccccc}
\hline & $\begin{array}{c}P_{\text {idle }} \\
(m W)\end{array}$ & $\begin{array}{c}P_{\text {sta }} \\
(m W)\end{array}$ & $\begin{array}{c}E_{\text {sw }} \\
(m J)\end{array}$ & $\begin{array}{c}t_{s w} \\
(m s)\end{array}$ & $f_{\text {cir }}$ \\
\hline PXA270 & 15.4 & 0.163 & 0.24 & 69.575 & 0.2211 \\
XSale & 40 & 0.8 & 0.5 & 85 & 0.2633 \\
\hline
\end{tabular}

TABLE III

POWER PARAMETERS FOR PXA270 AND XSCALE

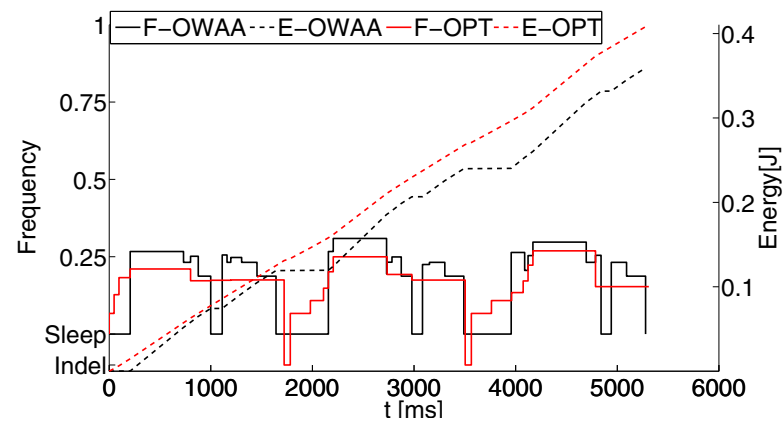

(a) OWAA vs DVS-OPT

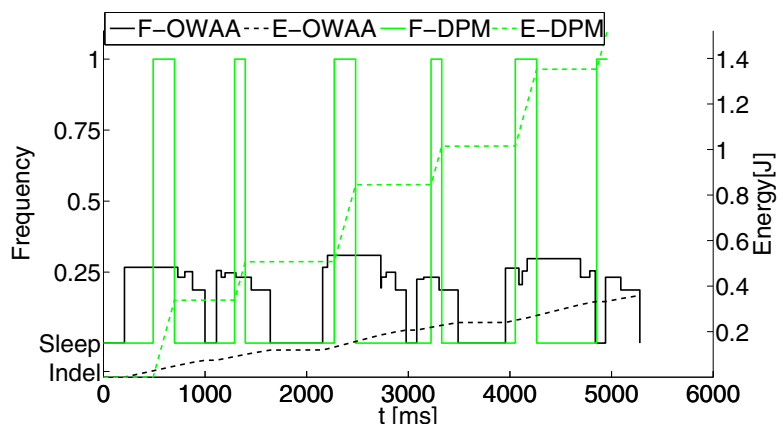

(b) OWAA vs DPM-DNC

Fig. 5. Frequency Schedules and Energy Consumption

pared the energy consumption with the following algorithms:

-DPM-DNC: A latest online DPM algorithm for aperiodic tasks proposed in [13] which minimizes the energy consumption with workload prediction using dynamic counters.

-DVS-AVR: The frequency decisions are performed by $\max \left(f_{\min }, \sum_{e_{i}: a_{i} \leq t \leq d_{i}} \frac{C}{D}\right)$, where $a_{i}$ and $d_{i}$ are respectively the arrival time and absolute deadline of the event $e_{i}$, and $\mathrm{C}$ and $\mathrm{D}$ are the worst-case execution time and the relative deadline [5].

-DVS-OPT: It makes the scheduling decision at time $t$ by processing the event with the earliest deadline at speed $\max \left(f_{\text {min }}, \max _{e_{j}} \sum_{e_{i}: a_{i} \leq t, d_{i} \leq d_{j}} \frac{C_{i}(t)}{d j-t}\right)$, where the event $e_{i}$ is associated with arrival time $a_{i}$ and absolute deadline $d_{i}$, and $C_{i}(t)$ is the remaining execution time at time $t[5]$.

-OWAA: Power management scheme base on the optimal workload-aware algorithm (OWAA) proposed in Section V.

\section{B. Simulation Result}

Qualitative evaluation: We conduct the qualitative examinations on XScale processor for stream $S_{1}$. The experiment runs for five seconds. Fig. 4 shows the event completion time and

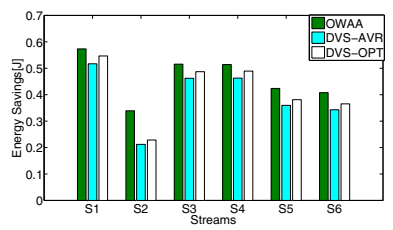

(a) PXA270

Fig 6 . Energy Savings Cor

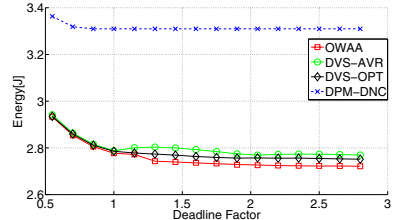

(a) PXA270

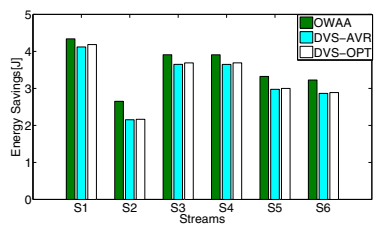

(b) XScale

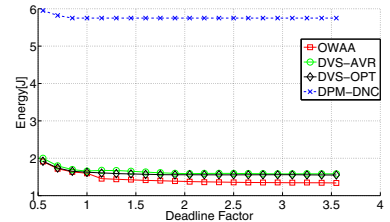

(b) XScale
Fig. 7. Energy Consumption of Different Deadline Settings Combining DVS and DPM

its deadline. The solid line $T F$ and dashed line $T D$ show the event completion curve and its deadline curve, respectively. As it can be seen, all events are completed before their deadlines.

Fig. 5 depicts frequency schedule and the resulting accumulative energy consumption produced by our scheme and DVS-OPT scheme where the prefix F- and E- represent the frequency schedule and the accumulative energy consumption of the schemes. As it can be seen, using the DVS-OPT scheme, the processor turns from idle state to active mode right after the new event arrives and runs with a low frequency. As the counterpart, our scheme first performs an appropriate delay to reduce the energy consumption and then selects a higher frequency to execute the tasks. From the accumulative energy consumption curves of both schemes (Fig. 5(a)), one can find out that our scheme exhibits better power efficiency than the DVS-OPT scheme during the entire time span.

Fig. 5(b) compares our scheme and the DPM-DNC scheme. Using DPM-DNC, the processor executes at the maximum speed to shorten the execution time and greedily explore the idle interval to switch the processor into sleep mode. However, the accumulative energy consumption curves in Fig. 5(b) show that doing this is actually suboptimal from the system point of view, due to the high dynamic power consumption. Unlike DPM-DNC, our scheme considers the trade-offs between dynamic and static power consumptions and controls the processor to run at an appropriate lower frequency according to the current workload, resulting in a lower overall power consumption.

Quantitative comparison: First, we consider the overall energy consumption and compare the proposed scheme with the three existing schemes. We run the simulation for 20 seconds and set the deadline factor to $\eta=1.6$. Fig. 6 shows the energy savings w.r.t DPM-DNC ${ }^{1}$ on PXA270 and XScale processors for streams listed in Tab. I. As depicted in Fig. 6, the proposed OWAA scheme outperforms the other three schemes in both devices. On average, for the PXA270 processor, our scheme

\footnotetext{
${ }^{1}$ energy savings of $x$ policy w.r.t $y$ policy: EnergySaving $=\mathrm{PC}(\mathrm{y})-\mathrm{PC}(\mathrm{x})$,
} where $\mathrm{PC}(\mathrm{x})$ is power consumption of $x$ 
achieves $462.3 \mathrm{~mJ}$ and $45.9 \mathrm{~mJ}$ energy savings w.r.t DPMDNC and DVS-OPT respectively. For the XScale processor, our scheme saved $3559.8 \mathrm{~mJ}$ and $290.2 \mathrm{~mJ}$ respectively w.r.t DPM-DNC and DVS-OPT.

In the next set of experiments, we compares the overall power consumption of the four schemes running on both devices with varying deadline factor $\eta$. The simulation time is again $20 \mathrm{~s}$ and stream $S_{1}$ is used as the input. Fig. 7 shows the results. As it can be seen, our online scheme that combines both DVS and DPM achieves the best results. For a small deadline factor $(\eta \leq 1)$, the number of unprocessed events that our algorithm can aggregate is limited and the opportunity for DPM is low. In this case, the contribution of energy savings mainly comes from the dynamic frequency scaling. We can see from Fig. 7 that OWAA can still exhibit higher energy gain compared to the other three schemes. With increasing deadline factor, OWAA can achieve further static power saving by combining the advantages of DVS and DPM. When the deadline factor $\eta$ is large enough, power consumption curves of all schemes become flat. The energy consumption on PXA270 stays constantly at $2.72 \mathrm{~J}, 2.75 \mathrm{~J}$, $2.77 \mathrm{~J}$, and $3.31 \mathrm{~J}$, using OWAA, DVS-OPT, DVS-AVR, and DPM-DNC scheme, respectively. One observation from Fig. 7 is that the performance gap between DPM-DNC and the other three algorithms is relatively small for the case of PXA270. The main reason is that the power consumption function of PXA270 is almost linear to the frequency (recall that $\gamma=1.26$ ) and the trade-off between DVS and DPM is not significant. For the case of XScale, the power consumptions figures are $1.33 J, 1.55 J, 1.58 J$, and $5.75 J$ for OWAA, DVSOPT, DVS-AVR, and DPM-DNC schemes respectively. Our OWAA scheme saves about $14.19 \%$ power consumption w.r.t DVS-OPT on XScale.

\section{CONCLUSION}

This paper presents an efficient framework for online energy optimization that considers the interplay of DVS and DPM. The core of the framework is an online algorithm that dynamically determines the optimal processor speed and mode transitions based on the runtime workload. Our algorithm is in $O(n)$ complexity and it takes into account the breakeven time and available frequency bounds of the processor.We also provides a guideline for configuring the system buffer size based on a feasibility analysis.Experimental results with specifications from two commercial processors show that our framework outperforms existing online power management approaches in the literature.

\section{REFERENCES}

[1] H. Aydin, R. Melhem, D. Mosse, and P. Mejia-Alvarez. Dynamic and aggressive scheduling techniques for power-aware real-time systems. In RTSS 2001: Proceedings of the 2001 22th IEEE Real-Time Systems Symposium, pages $95-105,2001$.

[2] N. Bansal, T. Kimbrel, and K. Pruhs. Dynamic speed scaling to manage energy and temperature. In Proceedings of the 2004 45th Annual IEEE Symposium on Foundations of Computer Science, pages 520 - 529, 2004.
[3] K. Bhatti, C. Belleudy, and M. Auguin. Power management in real time embedded systems through online and adaptive interplay of dpm and dvfs policies. In Proceedings of the 2010 IEEE/IFIP 8th International Conference on Embedded and Ubiquitous Computing, pages $184-191$, 2010.

[4] J.-J. Chen and T.-W. Kuo. Procrastination determination for periodic real-time tasks in leakage-aware dynamic voltage scaling systems. In ICCAD 2007: Proceedings of the 2007 IEEE/ACM International Conference on Computer-Aided Design, pages 289-294, 2007.

[5] J.-J. Chen, N. Stoimenov, and L. Thiele. Feasibility analysis of on-line dvs algorithms for scheduling arbitrary event streams. In RTSS 2009: Proceedings of the 2009 30th IEEE Real-Time Systems Symposium, pages $261-270,2009$.

[6] V. Devadas and H. Aydin. Dfr-edf: A unified energy management framework for real-time systems. In Proceedings of the 2010 16th IEEE Real-Time and Embedded Technology and Applications Symposium (RTAS), pages $121-130,2010$.

[7] V. Devadas and H. Aydin. On the interplay of voltage/frequency scaling and device power management for frame-based real-time embedded applications. IEEE Transactions on Computers, 61(1):31 -44, 2012.

[8] G. Dhiman and T. Rosing. Dynamic power management using machine learning. In ICCAD 2006: Proceedings of the 2006 IEEE/ACM International Conference on Computer-Aided Design, pages 747 -754, 2006.

[9] K. Huang, L. Santinelli, J.-J. Chen, L. Thiele, and G. Buttazzo. Adaptive power management for real-time event streams. In Proceedings of the 2010 15th Asia and South Pacific Design Automation Conference(ASP$D A C)$, pages $7-12,2010$.

[10] K. Huang, L. Santinelli, J.-J. Chen, L. Thiele, and G. Buttazzo. Applying real-time interface and calculus for dynamic power management in hard real-time systems. Real-Time Systems, 47:163-193, 2011. 10.1007/s11241-011-9115-z.

[11] C.-M. Hung, J.-J. Chen, and T.-W. Kuo. Energy-efficient real-time task scheduling for a dvs system with a non-dvs processing element. In RTSS 2006: Proceeding of the 2006 27th IEEE International Real-Time Systems Symposium, pages 303 -312, 2006.

[12] R. Jejurikar, C. Pereira, and R. Gupta. Leakage aware dynamic voltage scaling for real-time embedded systems. In DAC 2004: Proceedings of 2004 41st ACM/IEEE Design Automation Conference, pages 275 -280, 2004.

[13] K. Lampka, K. Huang, and J.-J. Chen. Dynamic counters and the efficient and effective online power management of embedded real-time systems. In CODES+ISSS 2011: Proceedings of the 9th IEEE/ACM International Conference on the Hardware/Software Codesign and System Synthesis, pages $267-276,2011$.

[14] J. Le Boudec and P. Thiran. Network Calculus: A Theory of Deterministic Queuing Systems for the Internet. Springer, 2001.

[15] J.-Y. Le Boudec. Application of network calculus to guaranteed service networks. IEEE Transactions on Information Theory, 44(3):1087-1096, may 1998.

[16] S. Maxiaguine, A. Chakraborty and L. Thiele. Dvs for buffer-constrained architectures with predictable qos-energy tradeoffs. In CODES+ISSS '05: Proceedings of the 2005 IEEE/ACM International Conference on Hardware/Software Codesign and System Synthesis, pages $111-116$, 2005.

[17] S. Perathoner, K. Lampka, N. Stoimenov, L. Thiele, and J.-J. Chen. Combining optimistic and pessimistic dvs scheduling: An adaptive scheme and analysis. In ICCAD 2010: Proceedings of the 2010 IEEE/ACM International Conference on Computer-Aided Design, pages $131-138,2010$.

[18] Marvell PXA270. http://www. marvell.com/application-processors/pxa-family

[19] L. Thiele, S. Chakraborty, and M. Naedele. Real-time calculus for scheduling hard real-time systems. In Proceedings of the 2000 IEEE International Symposium on Circuits and Systems, volume 4, pages 101 -104 vol.4, 2000.

[20] E. Wandeler and L. Thiele. Real-Time Calculus (RTC) Toolbox. http: //www. mpa. ethz.ch/Rtctoolbox, 2006.

[21] R. Xu, D. Mossé, and R. Melhem. Minimizing expected energy consumption in real-time systems through dynamic voltage scaling. ACM Trans. Comput. Syst., 25(4), 2007.

[22] F. Yao, A. Demers, and S. Shenker. A scheduling model for reduced cpu energy. In Proceedings of 36th Annual Symposium on Foundations of Computer Science, pages $374-382,1995$. 